\title{
Alterations in IQGAP1 expression and localization in colorectal carcinoma and liver metastases following oxaliplatin-based chemotherapy
}

\author{
DEBORAH ROTOLI ${ }^{1,2 *}$, MANUEL MORALES $^{3,4 *}$, MARÍA DEL CARMEN MAESO $^{5}$, \\ MARÍA DEL PINO GARCÍA ${ }^{6}$, RICARDO GUTIERREZ ${ }^{7}$, FRANCISCO VALLADARES ${ }^{7}$, JULIO ÁVILA ${ }^{1}$, \\ LUCIO DÍAZ-FLORES ${ }^{7}$, ALI MOBASHERI $^{8,9}$ and PABLO MARTÍN-VASALLO ${ }^{1}$
}

\begin{abstract}
${ }^{1}$ Laboratory of Developmental Biology, UD-Biochemistry and Molecular Biology and Centre for Biomedical Research of The Canary Islands, University of La Laguna, 38206 La Laguna, Canary Islands, Spain; ${ }^{2}$ National Research Council, Institute of Endocrinology and Experimental Oncology, I-80131 Naples, Italy; ${ }^{3}$ Service of Medical Oncology, University Hospital Nuestra Señora de Candelaria, 38010 Santa Cruz de Tenerife; ${ }^{4}$ Medical Oncology, Hospiten Rambla, 38001 Santa Cruz de Tenerife; ${ }^{5}$ Service of Pathology, University Hospital Nuestra Señora de Candelaria,

38010 Santa Cruz de Tenerife; ${ }^{6}$ Department of Pathology, Hospiten Rambla, 38001 Santa Cruz de Tenerife;

${ }^{7}$ Department of Pathology, School of Medicine, University of La Laguna, 38201 La Laguna, Canary Islands, Spain;

${ }^{8}$ Department of Veterinary Preclinical Sciences, School of Veterinary Medicine, Faculty of Health and Medical Sciences, University of Surrey, Guildford GU2 7XH, UK; ${ }^{9}$ Center of Excellence in Genomic Medicine Research,

King Fahd Medical Research Center, Faculty of Applied Medical Sciences,

King Abdulaziz University, Jeddah 21589, Saudi Arabia
\end{abstract}

Received July 21, 2016; Accepted October 11, 2016

DOI: $10.3892 / 01.2017 .6525$

\begin{abstract}
IQGAP1 is a scaffolding protein that serves a key role in cell dynamics by integrating internal and external stimuli to distinct signal outputs. Previous studies have identified several genes that are significantly up- or downregulated in the peripheral white cells (PWCs) of patients with colorectal adenocarcinoma (CRC), who underwent oxaliplatin-based chemotherapy (CT). In addition, screening studies have reported that IQ-motif containing GTPase activating protein 1 (IQGAP1) transcriptional expression levels varied from 'off'
\end{abstract}

Correspondence to: Professor Pablo Martín-Vasallo, Laboratory of Developmental Biology, UD-Biochemistry and Molecular Biology and Centre for Biomedical Research of The Canary Islands, University of La Laguna, Avenue Astrofísico Sánchez s/n, 38206 La Laguna, Canary Islands, Spain

E-mail: pmartin@ull.es

*Contributed equally

Abbreviations: AJ, adherens junction; APC, adenomatous polyposis coli; CRC, colorectal adenocarcinoma; CT, chemotherapy; EMT, epithelial mesenchymal transition; FOLFOX, folinic acid, leucovorin, 5-fluorouracil and oxaliplatin; IQGAP1, IQ-motif containing GTPase activating protein 1; MAPK, mitogen-activated protein kinase; PCNA, proliferating cell nuclear antigen; PWCs, peripheral white cells

Key words: colorectal cancer, oxaliplatin, IQGAP1, scaffold protein to 'on' following oxaliplatin CT. In order to determine if variations previously described in PWCs are able to be observed at the protein level in tumors and in metastases following CT, the present study performed an immunohistochemical analysis of IQGAP1 in CRC and primary metastases. IQGAP1 expression was observed in the nuclear envelope and in lateral cell membranes and cytoplasm in normal colon tissue. However, in tumor tissue, cells exhibited a diffuse pattern, with variable expression levels of staining in the nuclear membrane and cytoplasm, with the highest expression intensity observed at the invasive front. In healthy and metastasized liver tissue and in the metastases themselves, expression levels varied from cell to cell from no expression to a high level. In the majority of cells, IQGAP1 co-localized with microtubules at the cytoplasmic face of the nuclear envelope. Strong positive expression was observed in areas of the lesion where cells were detaching from the lesion into the lumen. Despite the homogeneous IQGAP1 staining pattern observed in healthy colon tissue sections, CRC demonstrated heterogeneity in staining, which was more marked in metastasized liver tissue resected following CT. However, the most notable findings were the observed effects on the cellular and subcellular distribution and its implications for cancer biology. These results suggest that IQGAP1 may be a putative biomarker, a candidate for clinical diagnostics and a potential novel target for anti-cancer therapeutics.

\section{Introduction}

Folinic acid, leucovorin, 5-fluorouracil and oxaliplatin (FOLFOX)-based chemotherapy (CT) is widely used in the 
treatment of colorectal adenocarcinoma (CRC). The addition of the platinum-containing compound oxaliplatin to the standard adjuvant treatment with 5-fluorouracil plus leucovorin enhances the efficacy of CT, doubling the response rate and prolonging progression-free survival of patients with stage II-III CRC $(1,2)$. Despite positive results obtained from this chemotherapeutic regimen, unwanted side effects are significant and require monitoring, as they affect, to various degrees, the quality of patient.

In an attempt to identify biomarkers that may predict the onset of these secondary effects, a recent study investigated the differential gene expression of peripheral leukocytes in patients with CRC prior to and following 3 cycles of oxaliplatin-based CT (3). The study identified 502 differentially-expressed genes that were significantly up- or downregulated in the peripheral white cells (PWCs) of patients following CT treatment (3).

To determine whether the changes in gene expression observed in PWCs may be detected in tumors following the administration of adjuvant $\mathrm{CT}$, the present study performed immunohistochemical analysis of a selected number of genes that had previously been identified in the differential transcriptome profile (4). The study observed that one gene among four, whose transcriptional expression levels varied from 'off' to 'on', was that coding for IQ-motif containing GTPase activating protein 1 (IQGAP1) (3).

IQGAP1 is a ubiquitously expressed scaffold protein, which contains a number of protein interaction domains (5). The protein interacts with cell adhesion molecules, components of the cytoskeleton, and various signaling molecules to regulate cell motility and morphology, cell cycle and other cellular functions $(5,6)$. By regulating its binding partners, IQGAP1 integrates a number of signaling pathways, several of which contribute to tumorigenesis. For example, IQGAP1 is associated with actin dynamics by direct binding to actin or indirect regulation via cell division cycle 42/Rac1 (7). Through its polyproline protein-protein domain (WW domain), the protein modulates the mitogen-activated protein kinase (MAPK) pathway, which is associated with cell cycle control (8). Therefore, IQGAP1 links MAPK signaling (for example, decisions regarding cell fate) to the cytoskeleton or cellular adhesion, with important implications for cancer.

Furthermore, interactions of IQGAP1 with extracellular signal-regulated kinases $1 / 2$ and MEK1/2 may lead to activation of the MAPK signaling pathway, thus modulating cell differentiation and proliferation (8). Therefore, IQGAP1 serves pivotal roles in several cellular functions, including control of polarization, cell adhesion, migration, proliferation and angiogenesis.

Numerous immunohistochemical studies have demonstrated that IQGAP1 is overexpressed in several forms of cancer and an aberrant membrane accumulation is observed, particularly at the invasive front $(9,10)$. Higher expression and altered localization of IQGAP1 from the cytoplasm to the membrane correlates with tumor grade and poor prognosis (11). The presence of IQGAP1 in the cell membrane may decrease adherens junction (AJ) function, favoring dissociation of the tumor cells (12).

The present study reports the alteration of IQGAP1 expression in CRC and liver metastases following CT administration at the protein level by performing an immunohistochemical analysis.

\section{Patients and methods}

Patients. The study was approved by the Ethics Committee of La Laguna University (La Laguna, Canary Islands, Spain) and the Ethical Committee of Nuestra Señora de Candelaria University Hospital (HUNSC; Santa Cruz de Tenerife, Canary Islands, Spain). All patients were treated at University Hospital of the Nuestra Señora de Candelaria, Santa Cruz de Tenerife, Spain between May 2007 and September 2015 and provided informed consent for the diagnosis and research of tissue specimens prior to enrollment in the study. All patients had colonic cancer and liver metastasis, which had been treated with FOLFOX-CT, (day 1, oxaliplatin $100 \mathrm{mg} / \mathrm{m}^{2}$ iv over $2 \mathrm{~h}$; leucovorin calcium $400 \mathrm{mg} / \mathrm{m}^{2}$ iv over $2 \mathrm{~h}$; followed by 5-fluorouracil $400 \mathrm{mg} / \mathrm{m}^{2}$ iv bolus and by 5 -fluorouracil $2400 \mathrm{mg} / \mathrm{m}^{2}$ iv over $46 \mathrm{~h}$; every 14 days). All patients received CT following resection of the primary tumor. Therefore, the primary tumors were CT-naïve, while the liver metastases were CT-treated.

Tumor tissue. Paraffin-embedded tissue samples from 15 patients ( 7 males and 8 females), ensuring patient's anonymity, and the corresponding clinical data were obtained from the reference medical areas of HUNSC.

Antibodies. The following antibodies were used: Rabbit anti-human polyclonal antibody against IQGAP1 (dilutions, 1:500 for immunohistochemistry and 1:250 for immunofluorescence; cat. no. ABT186; EMD Millipore, Billerica, MA, USA); mouse monoclonal antibody clone PC10 against anti-proliferating cell nuclear antigen (PCNA; dilution, 1:100; cat. no. 1486772; Roche Diagnostics GmbH, Mannheim, Germany); mouse monoclonal anti-human cluster of differentiation (CD)34 class II clone QBEnd 10 (ready-to-use; cat. no. IR632; Dako; Agilent Technologies GmbH, Waldbronn, Germany); and mouse monoclonal anti- $\beta$ tubulin (dilution, 1:150; cat. no. sc-101527; Santa Cruz Biotechnology, Inc., Dallas, TX, USA). Secondary antibodies: Biotin-conjugated anti-rabbit secondary antibody (dilution, 1:300; cat. no. 31820; Pierce; Thermo Fisher Scientific, Inc., Waltham, MA, USA); fluorescein isothiocyanate (FITC)-conjugated goat polyclonal antibody against rabbit IgG (cat. no. F9887; Sigma-Aldrich, Merck Merck Millipore, Darmstadt, Germany; dilution, 1:200); goat polyclonal antibody against mouse IgG (DyLight ${ }^{\circledR} 650$; cat. no. ab97018; Abcam, Cambridge, UK; dilution, 1:100).

Immunohistochemistry. Immunoperoxidase staining of paraffin-embedded tissue sections (fixed in 10\% formalin, for $48-72 \mathrm{~h}$ at $4^{\circ} \mathrm{C}$ ) was performed using the avidin-biotin reaction. Briefly, $5-\mu \mathrm{m}$-thick tissue sections were deparaffinized in xylene and hydrated in a graded series of alcohol baths. Heat-induced epitope retrieval was achieved by heating samples in sodium citrate buffer $\left(\mathrm{pH} \mathrm{6.0)}\right.$ at $120^{\circ} \mathrm{C}$ for $10 \mathrm{~min}$ in an autoclave. Once non-specific sites were blocked with $5 \%$ non-fat dry milk in TBS for $1 \mathrm{~h}$ at room temperature, endogenous biotin was blocked using the Avidin/Biotin Blocking kit (Vector Laboratories Inc., Burlingame, CA, USA). The primary antibody against IQGAP1 was applied (dilution, 1:500) to 


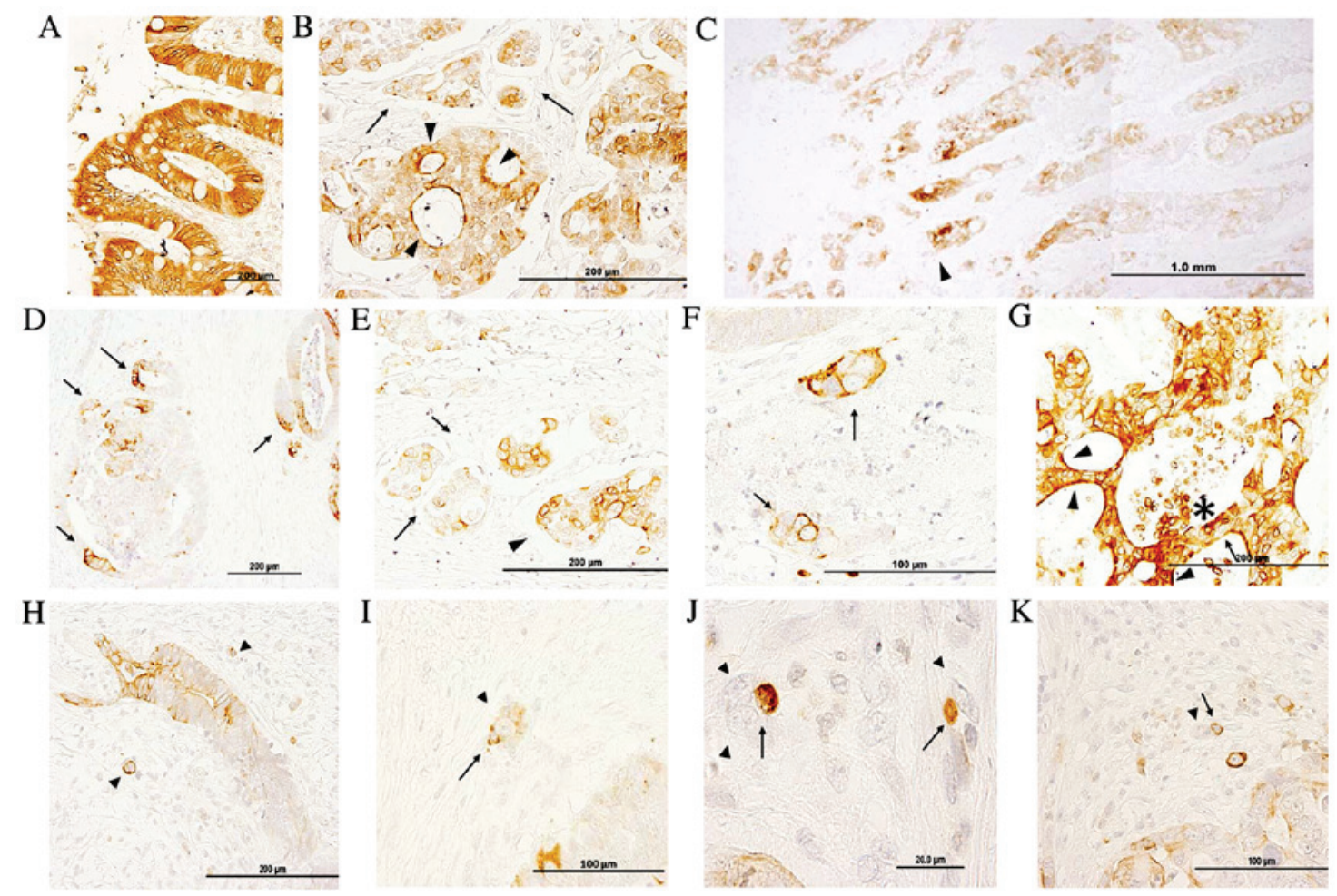

Figure 1. Immunohistochemical analysis of IQGAP1 protein expression in normal colon and colon adenocarcinoma tissue sections. (A) In normal colon tissue, positive staining was observed in the nuclear membrane, and in the lateral cell membrane and cytoplasm. (B) Tumor exhibiting heterogeneous IQGAP1 localization in the cytoplasm, nuclear membrane and plasma membrane. Arrowheads point to intense apical cell membrane staining, while arrows indicate budding tumor cells. (C) Low magnification of a CRC tumor section demonstrating a higher grade of IQGAP1 expression at the invasive front (arrowhead). (D) Tumor glands exhibiting a polarized positive signal. Cells located at the invasive front exhibited a strong membranous staining (arrows). (E) Tumor budding at the invasive front. Arrows identify open lumen lymphatic ducts with disseminated cancer cell nests. Nests demonstrated variable staining in the cytoplasm and nuclear envelope. Arrowhead points to the invasive lesion. (F) Cancer cell clusters with intense membranous staining (arrows) and lighter staining in cytosol. (G) Intense immunopositivity in a tumor gland exhibiting diffused localization (cytoplasm and nuclear membrane). Note the strong apical localization (arrowheads). Arrow points to unstained cells intermixed with IQGAP1 ${ }^{+}$cells. (H-K) Arrowheads identify budding tumor cells. Arrows point to IQGAP1 ${ }^{+}$immune cells associated with budding cells. Micrographs in the assembled figure were selected from different CRC cases. IQGAP1, IQ-motif containing GTPase activating protein 1; CRC, colorectal adenocarcinoma.

slides over night at $4^{\circ} \mathrm{C}$. To block endogenous peroxidase activity, the slides were incubated with $3 \%$ hydrogen peroxidase in methanol for $15 \mathrm{~min}$. Biotin-conjugated anti-rabbit secondary antibody (dilution, 1:300; Pierce; Thermo Fisher Scientific, Inc., Waltham, MA, USA) was incubated for $2 \mathrm{~h}$ at $37^{\circ} \mathrm{C}$, and ABC Peroxidase Staining kit (Thermo Fisher Scientific, Inc.) was used to amplify the specific antibody staining. 3,3'-diaminobenzidine substrate concentrate (no. IHC-101F; Bethyl Laboratories Inc., Montgomery, TX, USA) was used to visualize immunohistochemical reactions. Samples incubated without primary antibodies were used as a negative control. Slides were counterstained with Harris hematoxylin solution DC (Panreac Química SLU, Barcelona, Spain) to visualize cell nuclei and mounted with Eukitt mounting medium (Panreac Química SLU). An optical light microscope (BX50; Olympus Corporation, Tokyo, Japan) was used to visualize the results of the immunostaining.

Image analysis and statistical analysis. To compile tables, two independent observers evaluated the specimens blindly. Staining intensities were graded as absent (-), weak (+), moderate $(++)$ or strong $(+++)$. These cut-offs were established by consensus between each investigator following an initial survey of the entire blind-coded material. In cases where scorings differed by more than one unit, the observers re-evaluated the specimens to reach a consensus. In other cases, means of the scorings were calculated.

Double immunofluorescence simultaneous staining. Following deparaffinization, hydration and a heat-induced epitope retrieval procedure (as described above), tissue sections were incubated simultaneously with a mixture of two distinct primary antibodies (rabbit anti-human polyclonal antibody against IQGAP1 and mouse monoclonal antibody anti-PCNA, anti-CD34 or anti- $\beta$-tubulin) overnight at $4{ }^{\circ} \mathrm{C}$. Slides were then incubated for $1 \mathrm{~h}$ at room temperature in the dark with a mixture of two secondary antibodies raised in different species and conjugated to two different fluorochromes [anti-rabbit fluorescein isothiocyanate-conjugated antibody (Sigma-Aldrich; Merck Millipore) and anti-mouse DyLight ${ }^{\circledR} 650$-conjugated antibody (Abcam)]. Slides were mounted with ProLong ${ }^{\circledR}$ Diamond Anti-fade Mountant with DAPI (Molecular Probes ${ }^{\circledR}$; Thermo Fisher Scientific, Inc.) to visualize cell nuclei. Slides were analyzed using a confocal microscope (FV1000; Olympus Corporation).

\section{Results}

IQGAPl expression and localization in healthy colon and CRC tissue sections. Fig. 1 demonstrates IQGAP1 immunoreactivity 
A

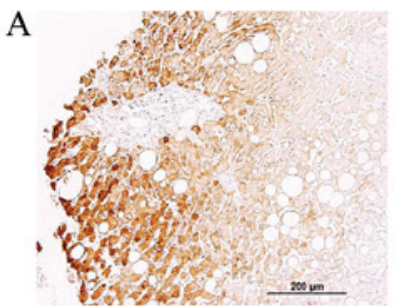

E

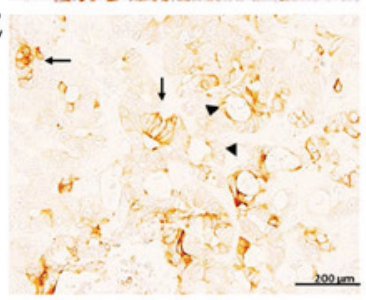

I .

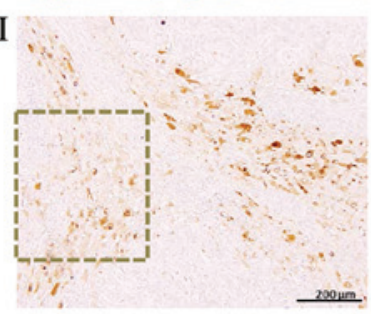

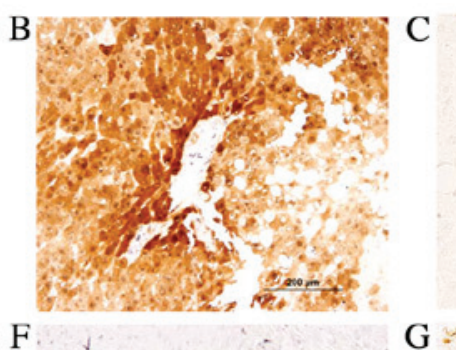

F

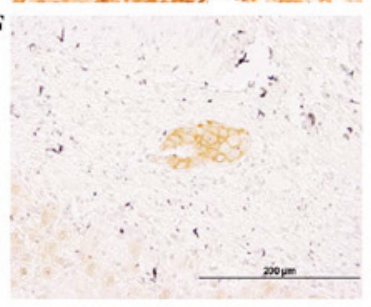

J

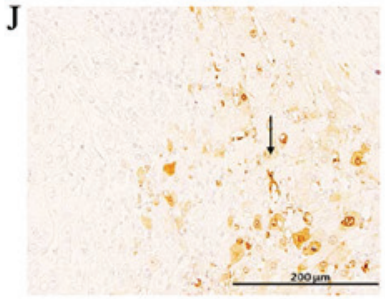

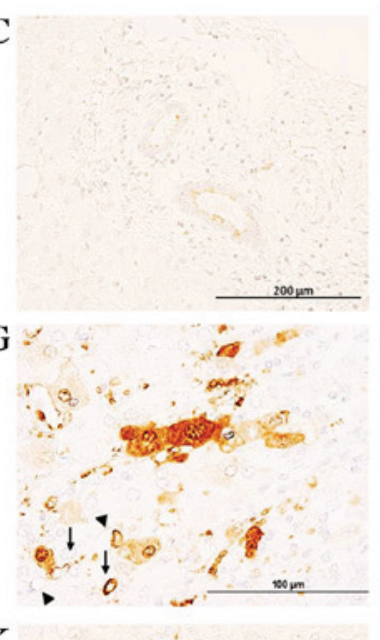

K

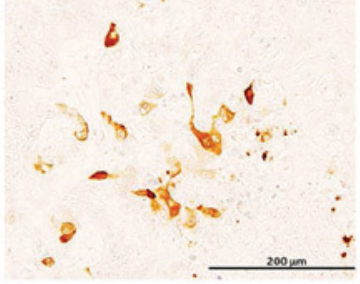

D

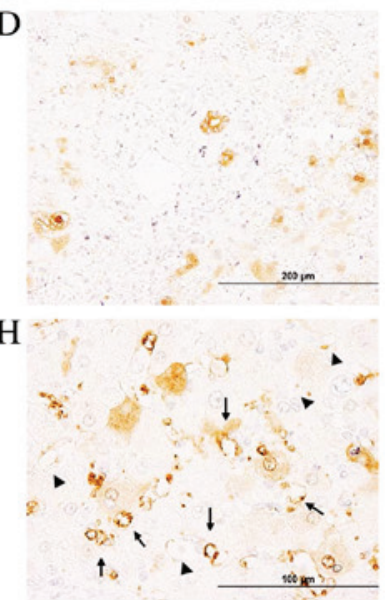

L.

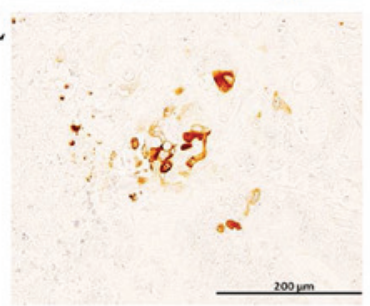

Figure 2. Immunohistochemical analysis of the expression of IQGAP1 in liver tissues. (A and B) Immunohistochemical staining demonstrated variable expression of IQGAP1 in hepatocytes in (A) healthy tissues and (B) a healthy area of metastasized liver tissue. Note that neighboring hepatocytes exhibited significantly different levels of expression. (C) Normal liver bile ducts with weak apical staining. (D) Metastasized liver portal trial with an intense positive membranous staining in bile ducts. (E) Metastases exhibited variable positive staining. Arrows point to malignant cells with strong membranous staining, while arrowheads point to areas of apical cell region staining. (F) Tumor glands demonstrated IQGAP1 ${ }^{+}$membranous staining. (G and $H$ ) Several microvessels exhibited a strong positive signal (arrows), while other vessels appeared negative (arrowheads). (I) Several stromal cells and microvessels located between lesions exhibited intense positive staining. (J) Higher magnification of the inset in (I); arrow points to an IQGAP1+ tumor-associated microvessel. (K and L) IQGAP1' tumor cells with tumor-associated fibroblast-like cells exhibited strong IQGAP1 staining. IQGAP1, IQ-motif containing GTPase activating protein 1.

in paraffin-embedded sections of normal colon and in healthy areas of adenocarcinoma sections. The signal revealed positive staining in enterocytes (nuclear membrane, cytoplasm, apical and lateral cell membrane) (Fig. 1A); the majority of cells were stained and only a few exhibited a lighter level of staining. IQGAP1 protein was localized in cytoplasm, nuclear envelope, cell junctions, plasma membrane and apical membrane, and this variable localization could be observed in the same structure concurrently (Fig. 1B and D-F). Within the tumor, tumor cells exhibited a diffuse pattern with a variable level of staining (from no staining to high intensity) in the nuclear envelope and in the cytoplasm. The intensity of staining was higher at the invasive front (Fig. 1C). Cancer cell nests exhibited variable positive perinuclear and cytoplasmic staining in the open-lumen lymphatic ducts located in the submucosa (Fig. 1B and E, black arrows). In Fig. 1D, the intense membranous IQGAP1 staining appeared polarized in several tumor glands, while the other cells of the lesion were negative or exhibited only weak reactivity. Thus, the expression pattern and localization of IQGAP1 protein in the CRC tissue sections was heterogeneous, both in the normal glandular epithelium and in tumor glands and nests. Strongly IQGAP1-positive cells were intermixed with unstained tumor cells within tumor lesions (Fig. 1G, black arrow); in several carcinoma cell clusters located in close proximity to tumor glands, a strong and diffuse membranous immunolocalization was observed, while nuclei were negative (Fig. 1F, black arrows). In several lesions, strong apical cell membrane staining was observed (arrowhead in Fig. 1G); in addition, strong IQGAP1 expression was observed in areas of the lesion where cells were detaching into the lumen (asterisk in Fig. 1G).

Fig. 1H-K shows representative images of budding tumor cells (arrowheads) either as small clusters of cells ( $<5$ cells) or as single cells. IQGAP1 expression was variable, with certain cells exhibiting a strong positive signal all along the cell membrane, while others were completely negative. The presence of IQGAP1 ${ }^{+}$immune cells (arrows) was associated with budding cells.

IQGAP1 expression and localization in healthy and metastasized liver tissue sections. In healthy liver and the healthy region of metastasized liver tissues, hepatocytes exhibited a clearly delimitated labeling in the plasma membrane, cytoplasm and nuclear envelope, exhibiting non-homogenous IQGAP1 ${ }^{+}$immunostaining, with neighboring hepatocytes demonstrating variable levels of expression (Fig. 2). Along the tissue sections, there were areas with no signal and areas exhibiting variable positive staining. The intensity of the signal was higher in the metastasized tissue sections compared with normal liver sections (Fig. 2A and B). Bile ducts exhibited weak apical staining in normal liver sections (Fig. 2C), while in metastasized liver sections they demonstrated intense membranous positive staining (Fig. 2D).

Metastases exhibited variable levels of IQGAP1 expression, and were heterogeneous in terms of intensity and localization (Fig. 2E-G). Positive immunostaining was observed in several 

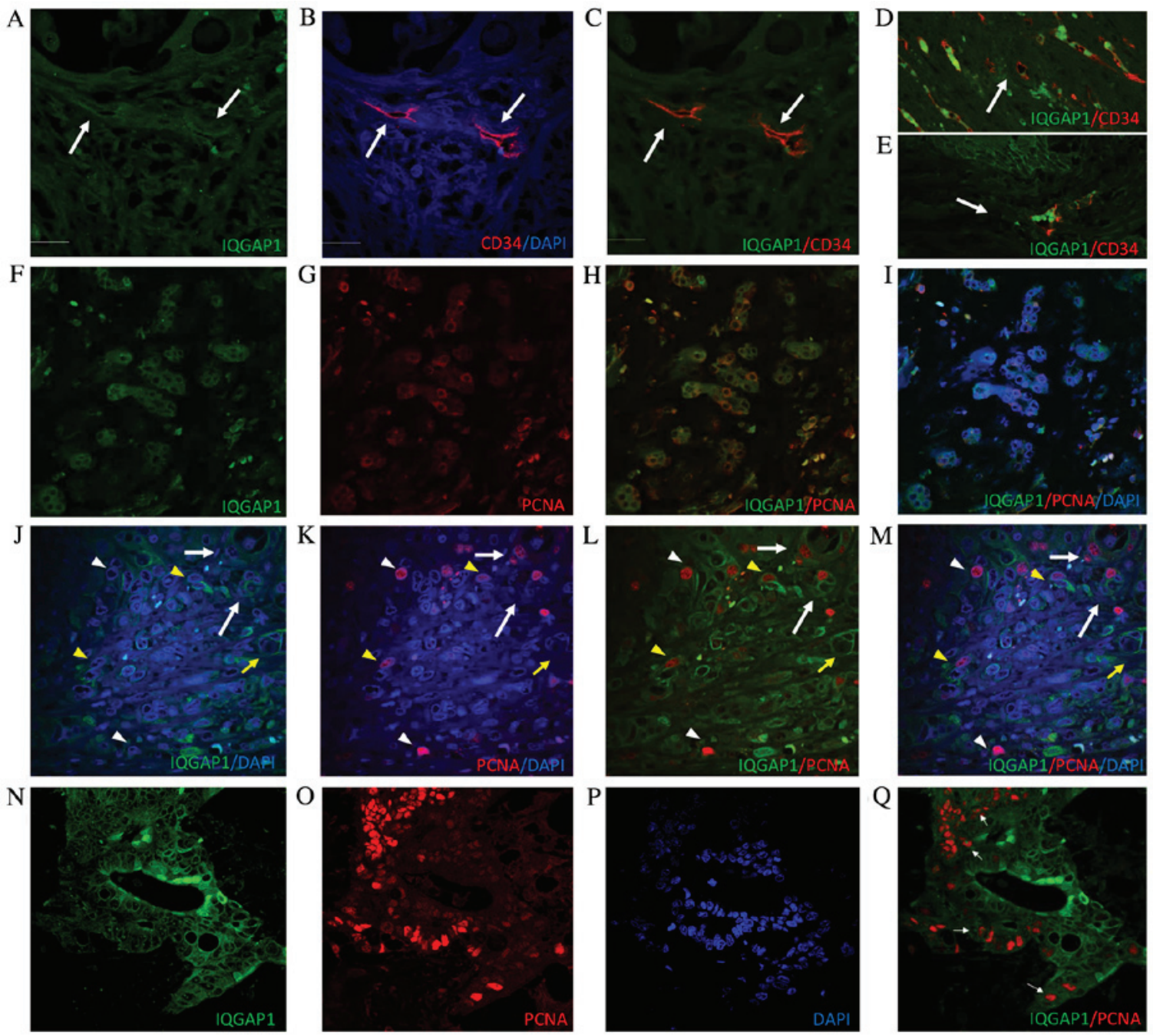

Figure 3. Confocal microscopic analysis of the protein expression by double immunostaining on paraffin-embedded tissue sections of CRC and metastasized liver for IQGAP1 (green) and CD34 (red) or PCNA (red) and DAPI. CRC tissue section; arrows point to tumor-associated vessels with positive staining for (A) IQGAP1 and (B) CD34 or (C) IQGAP1 and CD34 colocalization. Tumor-associated microvessels in (D) metastasized liver and in (E) CRC tissue sections IQGAP1 ${ }^{+} \mathrm{CD}_{3}{ }^{+}$microvessels are identified by arrows. (F-I) Metastasized liver double immunostaining for (F) IQGAP1 (green) and (G) PCNA (red). IQGAP1 and PCNA co-localized at the outer nuclear membrane in several cells of the tumor nests; (H) IQGAP1 and PCNA co-localization (I) in addition to DAPI (J-M) Metastasized liver section double immunostaining for IQGAP1 (green) and PCNA (red). Thin arrow points to a tumor cell cluster where IQGAP1 was observed along the cell membrane; note the presence of a PCNA ${ }^{+}$cell in the cluster. Yellow arrow points to an IQGAP1 ${ }^{+}$tumor-associated microvessel Thick white arrow points to tumor-associated blood vessel with PCNA labeled endothelial cells. Yellow arrowhead points to an IQGAP1 ${ }^{+}$PCNA ${ }^{+}$tumor cell. White arrowhead points to an IQGAP1- PCNA ${ }^{+}$tumor cell. (N-Q) CRC tissue section double immunostaining for (N) IQGAP1 (green) and (O) PCNA (red); (P) DAPI; (Q) IQGAP1 and PCNA colocalization. Note the positive IQGAP1 staining in the nucleoli of tumor cells (arrows). Scale bar, 50 $\mu$ m. IQGAP1, IQ-motif containing GTPase activating protein 1; CD, cluster of differentiation; PCNA, proliferating cell nuclear antigen; CRC, colorectal adenocarcinoma.

stromal cells (Fig. 2H-L) and in microvessels present in areas adjacent to the metastasis (Fig. $2 \mathrm{G}$ and $\mathrm{H}$ ). To assess the nature of these IQGAP1 ${ }^{+}$vessels, the vascular endothelium marker CD34 was used. Confocal analyses of tissue sections double immune-labeled for CD34 and IQGAP1 revealed co-localization of each protein in several vessels (Fig. 3A-E).

Tables I and II specify the IQGAP1 expression signatures observed in the tissue specimens.

To evaluate the proliferative activity of tumor cells, the proliferation marker PCNA was used in double immunofluorescence experiments on formalin-fixed, paraffin-embedded tissue sections. In several malignant cells forming tumor nests, IQGAP1 and PCNA protein exhibited partial co-localization. The distribution of intranuclear PCNA and IQGAP1 surrounding the outer membrane of the nucleus suggests that these cells were in the early $\mathrm{S}$ phase (Fig. 3F-I).
In Fig. 3J-M, IQGAP1 ${ }^{+}$staining was observed along the cell membrane of several tumor cells, and in tumor-associated blood and microvessels. Within the lesion, PCNA intensity and localization varied depending on the stage of the cell cycle in which the cells were. Notably, IQGAP1 $1^{+}$staining was frequently observed in cells in G1/S phase when PCNA begins to translocate from the cytoplasm to the nucleus (Fig. 3F-I), or in cells in early $\mathrm{S}$ phase when PCNA expression in the nucleus is weaker (Fig. 3J-M, yellow arrowhead). High levels of PCNA in the nucleus helps to identify late $\mathrm{S}$ phase cells; within these cells, no IQGAP1 ${ }^{+}$signal was observed (Fig. 3J-M, white arrowhead), except occasionally in the nucleolus (Fig. 3Q).

Given the key role of IQGAP1 in the regulation of the cytoskeleton, double immunofluorescence experiments were performed on CRC tissue sections to localize IQGAP1 and $\beta$-tubulin. As presented in Fig. 4, IQGAP1 co-localized with 
Table I. IQGAP1 expression and localization in healthy colon and CRC tissues.

\begin{tabular}{lcc}
\hline Cell type & Healthy colon & CRC \\
\hline Epithelial cells (Mucosae) & +++ & $+++/-$ \\
& $\mathrm{lm}, \mathrm{am}, \mathrm{n}, \mathrm{c}$ & $\mathrm{pm}, \mathrm{lm}, \mathrm{c}, \mathrm{n}$ \\
Stromal cells (Mucosae) & - & $++/-$ \\
Tumor cells & - & +++- \\
& & $\mathrm{pm}, \mathrm{c}, \mathrm{n}$ \\
Tumor associated stromal cells & - & $++/-$ \\
Immune cells & $?$ & +++ \\
Endothelial cells & ++ & ++ \\
& $\mathrm{n}$ & $\mathrm{n}, \mathrm{c}$ \\
Smooth muscle cells & ++ & - \\
& $\mathrm{n}$ & - \\
Neurons (Myenteric plexus) & ++ & - \\
Glial cells (Myenteric plexus) & ++ & \\
\hline
\end{tabular}

-, negative; ++, moderate; +++, high; ++/-, variable from moderate to absent; +++/-, variable from high to absent; ?, indeterminate staining; $\mathrm{lm}$, lateral membrane; am, apical membrane; pm, plasma membrane; c, cytoplasm; n, nucleus or nuclear envelope.

Table II. IQGAP1 expression and localization in healthy and colorectal adenocarcinoma metastasized liver.

\begin{tabular}{lcc}
\hline Cell type & Healthy liver & Metastasized liver \\
\hline Tumor cells & - & $++/-$ \\
& & $\mathrm{pm}, \mathrm{c}$ \\
Hepatocytes & $+++/-$ & $+++/$ \\
& $\mathrm{n}, \mathrm{c}$ & $\mathrm{n}, \mathrm{c}$ \\
Epithelial cells & + & ++ \\
(bile ducts) & $\mathrm{am}$ & $\mathrm{pm}$ \\
Tumor associated & - & ++ \\
stromal cells & &
\end{tabular}

-, negative; +, weak; ++, moderate; +++, high; ++/-, variable from moderate to absent; +++/-, variable from high to absent; am, apical membrane; pm, plasma membrane; c, cytoplasm; n, nucleus or nuclear envelope.

microtubules at the cytoplasmic face of the nuclear envelope in certain cells (arrows). However, in other cells, the pole of the cell was stained for IQGAP1 with no label for $\beta$-tubulin. Nucleolar staining was often observed throughout the fields of observation (Figs. 1-4).

\section{Discussion}

The present study aimed to study the expression of IQGAP1 in $\mathrm{CRC}$ and liver metastases following the administration of adjuvant $\mathrm{CT}$ using immunohistochemistry. In contrast to normal cells, in which IQGAP1 was homogeneously distributed, cancer cells presented variable expression patterns, ranging from no expression to whole cell, high level of expression
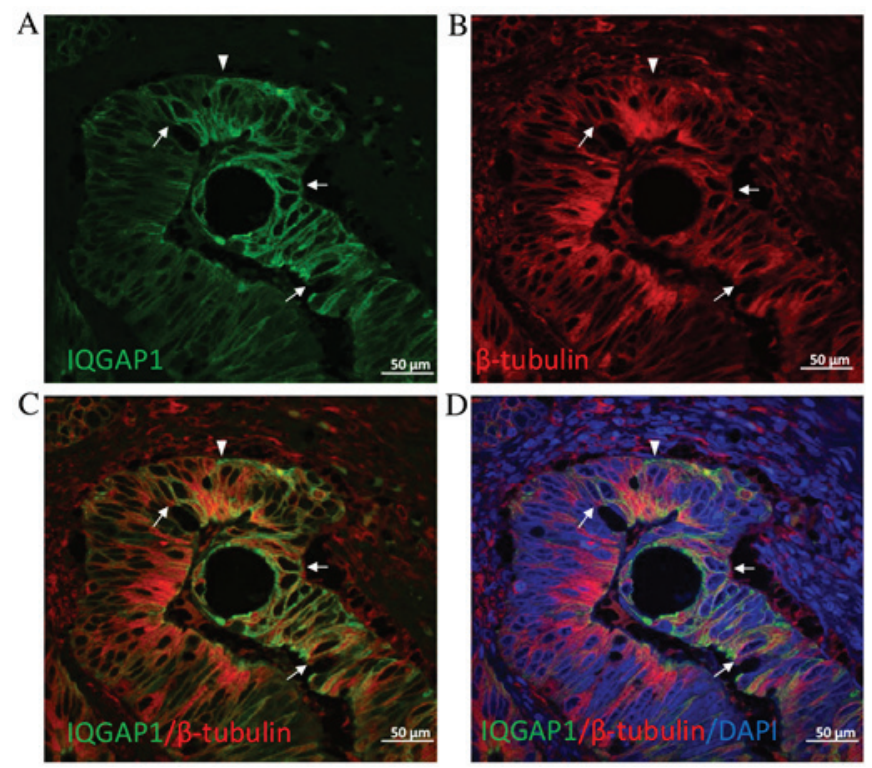

Figure 4. Confocal microscopic analysis of (A) IQGAP1 (green) and (B) $\beta$-tubulin (red) expression by double immunofluorescence staining on CRC tissue sections. (C) IQGAP1 and $\beta$-tubulin colocalization and with (D) the addition of the nuclear stain DAPI. In the majority of cells, IQGAP1 co-located with microtubules at the cytoplasmic face of the nuclear envelope (arrows). Note that several cells exhibited increased IQGAP1 expression at the plasma membrane (arrowhead) at areas with no (or extremely low) tubulin expression. IQGAP1, IQ-motif containing GTPase activating protein 1.

(Fig. 1A-C). As shown in Fig. 1C, maximum expression was observed at the growing front of the tumor gland indicating the expanding direction, which corresponded to the apical region of the expanding cell in which nuclei were in the opposite pole. Furthermore, double labeling by IQGAP1 and $\beta$-tubulin demonstrated that in certain tumor cells the association of each protein disappeared (Fig. 4), indicating clear points where epithelial to mesenchymal cell transition (EMT) occurs. This supports the role of IQGAP scaffold proteins in the regulation of membrane dynamics by coupling the cortical actin meshwork to microtubules via plus-end binding proteins and other proteins involved in intracellular signaling that, ultimately, results in modulation of more complex functions, including cytokinesis, cell migration, cell growth or survival. Specifically, IQGAP1 is observed in actin-dependent membrane structures (membrane ruffles implicated in cell locomotion and lamellipodia) interacting with the plus-end binding proteins cytoplasmic linker protein-170 and adenomatous polyposis coli (APC), tethering microtubules to the actin network $(13,14)$. Furthermore, in the nuclear envelope of certain tumor cells, co-expression of IQGAP1 protein and $\beta$-tubulin was observed (Fig. 4). This co-localization of IQGAP1 with the microtubule network at the cytoplasmic face of the nuclear envelope has previously been described by Johnson and Henderson (15) in MCF-7 (breast cancer epithelial cells), HT29 (colon cancer epithelial cells) and NIH3T3 (non-tumor embryonic fibroblasts) cell lines, and was also correlated with a possible role for IQGAP1 in cell polarization and migration events, in addition to cell cycle-associated nuclear envelope assembly/disassembly. Johnson and Henderson (15) suggested that interactions between IQGAP1 and microtubules may tether these cytoskeletal networks to perinuclear actin via the 
plus-end protein APC and/or interaction with other nuclear envelope proteins to regulate the microtubule organizing center and nuclear positioning for cell polarization during cell migration, a key process in tumorigenesis and carcinogenesis.

In the current study, increased expression and altered localization of IQGAP1 from the cytoplasm to the plasma membrane was observed in several tumor cells compared with healthy tissue (Figs. 1D, F and H, 2E and 4) may serve to decrease AJ stability, favoring dissociation of the tumor cells (12). IQGAP1 at the plasma membrane regulates the stability of the AJ complex, which is necessary for proper apical-basal polarity of epithelial cells that disappears during the EMT process (16).

In the present study, the co-localization of CD34 and IQGAP1 in several vessels (Fig. 3A-E) is indicative of a role for IQGAP1 in tumor vasculogenesis and/or in vascular invasion. Nakhaei-Nejad et al (17) studied the involvement of endothelial IQGAP1 in leukocyte transendothelial migration, and demonstrated, by RNAi silencing of IQGAP1 in human umbilical vein endothelial cells, that IQGAP1 and interendothelial junction-associated microtubules were involved in remodeling interendothelial junctions to facilitate lymphocyte diapedesis under physiological shear stress. Furthermore, Yamaoka-Tojo et al (18) reported that IQGAP1 is a novel vascular endothelial growth factor receptor 2 (VEGFR2) binding protein in quiescent endothelial cells and is important for the establishment of VE-cadherin-based cell-cell contacts, and suggested that IQGAP1 may function as a scaffold linking VEGFR2 to the $\beta$-catenin/VE-cadherin compound at the AJ (18).

PCNA is a DNA clamp that increases the processivity of DNA polymerase $\delta$ in eukaryotic cells, which is used as a marker of proliferating cells (19-21). The immunohistochemical experiments of the present study revealed that certain cells co-expressed IQGAP1 and PCNA in the cytoplasm, whereas others expressed IQGAP1 in the cytoplasm and PCNA in the nucleus, which is indicative of $\mathrm{S}$ phase (22). In addition, CD34 ${ }^{+}$ microvessels of CRC and metastatic sections co-express PCNA and IQGAP, while the majority of cancer cells do not contain intranuclear PCNA, though they may contain cytoplasmic, pointing to a quiescent phase while waiting for energy to be supplied in order for the carcinogenic process to progress (23).

As shown in Fig. 3F-M, during the synthesis of PCNA, PCNA and IQGAP1 are simultaneously expressed in the cytoplasm of cells within the liver. PCNA then enters into the nucleus and $\mathrm{S}$ phase of mitosis begins. A role for IQGAP1 in regulating early $\mathrm{S}$ phase replication events has been recently proposed. Johnson et al (23) identified nuclear localization of IQGAP1 in several mammalian cell lines. This nuclear localization was low in asynchronous cells, but was significantly increased in cells arrested in G1/S phase (23). The authors suggested that the protein enters the nucleus at G1/S phase and exits in late $\mathrm{S}$ phase. Furthermore, nuclear IQGAP1 was identified to function as part of a complex with replication protein A $32 \mathrm{kDa}$ subunit and PCNA, suggesting a functional role for IQGAP1 in the reinitiation of $\mathrm{S}$ phase following DNA replication arrest (23).

As observed in the current study, nucleoli staining is often observed throughout the field of microscopic observation, which is consistent with Bielak-Zmijewska et al (24). The authors observed the presence of IQGAP1 in mouse oocyte nuclei, forming a ring around the nucleolus only in transcriptionally active oocytes, but not in transcriptionally silent ones or in growing oocytes treated with the transcription inhibitor $\alpha$-amanitin, indicating an association between IQGAP1 expression in the nucleolus and RNA synthesis (24).

In conclusion, extremely few studies have analyzed IQGAP1 expression in colorectal tumors, and none have yet addressed IQGAP1 expression in CRC and its associated liver metastases. To the best of our knowledge, the present study is the first to provide a detailed analysis of IQGAP1 expression in CRC tissue sections and metastasized liver tissue samples, which were resected following oxaliplatin-based CT. Despite the homogeneous IQGAP1 staining pattern observed in healthy colon tissue sections, the CRC tissues exhibited heterogeneous expression (in terms of localization and intensity), which was more marked in the metastasized liver sections resected following CT treatment. However, more notable findings are the described effects over the cellular and subcellular distribution and its implications in the cancer biology. Therefore, IQGAP1 may be a novel cancer antigen, a surrogate biomarker of responses to $\mathrm{CT}$, a candidate for the development of new clinical diagnostics and a new target for anti-cancer therapeutics.

\section{Acknowledgements}

The present study was supported by grants from the Health Institute of Charles III, Spain (no. FIS PI11/00114) and Microscopy was funded by a joint grant from the Insular Council of Tenerife, Spain; the Ministry of Science and Technology, Spain; and the European Regional Development Fund 2003/2004, Belgium (no. IMBRAIN-FP7-REGPOT-2012-31 637).

\section{References}

1. André T, Boni C, Mounedji-Boudiaf L, Navarro M, Tabernero J, Hickish T, Topham C, Zaninelli M, Clingan P, Bridgewater J, et al: Oxaliplatin, fluorouracil, and leucovorin as adjuvant treatment for colon cancer. N Engl J Med 350: 2343-2351, 2004.

2. Xu R, Zhou B, Fung PC and Li X: Recent advances in the treatment of colon cancer. Histol Histopathol 21: 867-872, 2006.

3. Morales M, Ávila J, González-Fernández R, Boronat L, Soriano ML and Martín-Vasallo P: Differential transcriptome profile of peripheral white cells to identify biomarkers involved in oxaliplatin induced neuropathy. J Pers Med 4: 282-296, 2014.

4. Rotoli D, Morales M, Del Carmen Maeso M, Del Pino García M, Morales A, Avila J and Martín-Vasallo P: Expression and localization of the immunophilin FKBP51 in colorectal carcinomas and primary metastases, and alterations following oxaliplatin-based chemotherapy. Oncol Lett 12: 1315-1322, 2016.

5. Johnson M, Sharma M and Henderson BR: IQGAP1 regulation and roles in cancer. Cell Signal 21: 1471-1478, 2009.

6. White CD, Brown MD and Sacks DB: IQGAPs in cancer: A family of scaffold proteins underlying tumorigenesis. FEBS Lett 583: 1817-1824, 2009.

7. Erickson JW, Cerione RA and Hart MJ: Identification of an actin cytoskeletal complex that includes IQGAP and the Cdc42 GTPase. J Biol Chem 272: 24443-24447, 1997.

8. Roy M, Li Z and Sacks DB: IQGAP1 is a scaffold for mitogen-activated protein kinase signaling. Mol Cell Biol 25: 7940-7952, 2005 .

9. McDonald KL, O'Sullivan MG, Parkinson JF, Shaw JM, Payne CA, Brewer JM, Young L, Reader DJ, Wheeler HT, Cook RJ, et al: IQGAP1 and IGFBP2: Valuable biomarkers for determining prognosis in glioma patients. J Neuropathol Exp Neurol 66: 405-417, 2007. 
10. Nabeshima K, Shimao Y, Inoue T and Koono M: Immunohistochemical analysis of IQGAP1 expression in human colorectal carcinomas: Its overexpression in carcinomas and association with invasion fronts. Cancer Lett 176: 101-109, 2002.

11. Holck S, Nielsen HJ, Hammer E, Christensen IJ and Larsson LI: IQGAP1 in rectal adenocarcinomas: Localization and protein expression before and after radiochemotherapy. Cancer Lett 356: 556-560, 2015.

12. Takemoto H, Doki Y, Shiozaki H, Imamura H, Utsunomiya T, Miyata $\mathrm{H}$, Yano $\mathrm{M}$, Inoue $\mathrm{M}$, Fujiwara $\mathrm{Y}$ and Monden $\mathrm{M}$ : Localization of IQGAP1 is inversely correlated with intercellular adhesion mediated by e-cadherin in gastric cancers. Int J Cancer 91: 783-788, 2001.

13. Fukata M, Watanabe T, Noritake J, Nakagawa M, Yamaga M, Kuroda S, Matsuura Y, Iwamatsu A, Perez F and Kaibuchi K: Rac1 and Cdc42 capture microtubules through IQGAP1 and CLIP-170. Cell 109: 873-885, 2002.

14. Watanabe T, Wang S, Noritake J, Sato K, Fukata M, Takefuji M, Nakagawa M, Izumi N, Akiyama T and Kaibuchi K: Interaction with IQGAP1 links APC to Rac1, Cdc42, and actin filaments during cell polarization and migration. Dev Cell 7: 871-883, 2004.

15. Johnson MA and Henderson BR: The scaffolding protein IQGAP1 co-localizes with actin at the cytoplasmic face of the nuclear envelope: Implications for cytoskeletal regulation. Bioarchitecture 2: 138-142, 2012.

16. Noritake J, Watanabe T, Sato K, Wang S and Kaibuchi K: IQGAP1: A key regulator of adhesion and migration. J Cell Sci 118: 2085-2092, 2005.

17. Nakhaei-Nejad M, Zhang QX and Murray AG: Endothelial IQGAP1 regulates efficient lymphocyte transendothelial migration. Eur J Immunol 40: 204-213, 2010.
18. Yamaoka-Tojo M, Tojo T, Kim HW, Hilenski L, Patrushev NA, Zhang L, Fukai T and Ushio-Fukai M: IQGAP1 mediates VE-cadherin-based cell-cell contacts and VEGF signaling at adherence junctions linked to angiogenesis. Arterioscler Thromb Vasc Biol 26: 1991-1997, 2006.

19. Guzińska-Ustymowicz K, Pryczynicz A, Kemona A and Czyzewska J: Correlation between proliferation markers: PCNA, Ki-67, MCM-2 and antiapoptotic protein Bcl-2 in colorectal cancer. Anticancer Res 29: 3049-3052, 2009.

20. Bleau AM, Agliano A, Larzabal L, de Aberasturi AL and Calvo A: Metastatic dormancy: A complex network between cancer stem cells and their microenvironment. Histol Histopathol 29: 1499-1510, 2014.

21. Moldovan GL, Pfander B and Jentsch S: PCNA, the maestro of the replication fork. Cell 129: 665-679, 2007.

22. Connolly KM and Bogdanffy MS: Evaluation of proliferating cell nuclear antigen (PCNA) as an endogenous marker of cell proliferation in rat liver: A dual-stain comparison with 5-bromo-2'-deoxyuridine. J Histochem Cytochem 41: 1-6, 1993.

23. Johnson M, Sharma M, Brocardo MG and Henderson BR: IQGAP1 translocates to the nucleus in early S-phase and contributes to cell cycle progression after DNA replication arrest. Int J Biochem Cell Biol 43: 65-73, 2011.

24. Bielak-Zmijewska A, Kolano A, Szczepanska K, Maleszewski M and Borsuk E: Cdc42 protein acts upstream of IQGAP1 and regulates cytokinesis in mouse oocytes and embryos. Dev Biol 322: 21-32, 2008. 